\title{
The use of a retrograde fixed-angle intramedullary nail for tibiocalcaneal arthrodesis after severe loss of the talus
}

\author{
Kajetan Klos · Thomas Drechsel · Florian Gras • \\ Claudia Beimel · Andreas Tiemann • \\ Gunther O. Hofmann · Thomas Mückley
}

Received: 26 March 2009/Accepted: 19 August 2009/Published online: 10 September 2009

(C) Springer-Verlag 2009

\begin{abstract}
Tibiocalcaneal arthrodesis may be the only means of obtaining a painless and stable limb when there is loss of the talus. We present the early results of a prospective study on tibiocalcaneal arthrodesis using a latestgeneration retrograde intramedullary nail. In the period 2006-2007, nine patients underwent tibiocalcaneal arthrodesis with retrograde intramedullary nailing. Five of these patients had infection-related loss of the talus. SF-36, AOFAS ankle-hindfoot, and Mazur Ankle Arthrodesis scores were obtained pre-fusion, and at 6 weeks, 6 months and 1 year post-fusion. The patients were also followed up clinically and radiologically. Previous surgical procedures, chronic musculoskeletal problems and other comorbidities, and complications were recorded and analyzed. All patients
\end{abstract}

K. Klos $(\bowtie) \cdot$ F. Gras

Department of Trauma, Hand and Reconstructive Surgery,

University Hospital Jena, Erlanger Allee 101,

07743 Jena, Germany

e-mail: Kajetan.Klos@med.uni-jena.de

T. Drechsel

Department of Trauma and Reconstructive Surgery,

Berufsgenossenschaftliche Unfallkliniken

Bergmannstrost Halle (Saale), Halle, Germany

C. Beimel

Kiel, Germany

A. Tiemann

Department of Septic and Reconstructive Surgery,

Berufsgenossenschaftliche Unfallkliniken Bergmannstrost

Halle (Saale), Halle, Germany

G. O. Hofmann · T. Mückley

Department of Trauma, Hand and Reconstructive Surgery, University Hospital Jena, Berufsgenossenschaftliche

Unfallkliniken Bergmannstrost Halle (Saale), Halle, Germany were available for initial follow-up and were subjectively satisfied with their outcomes. Solid fusion was achieved and fully confirmed in nine cases. One subject died 8 weeks postoperatively of a pulmonary embolism. One patient had recurrent infection. At 1 year, only one patient still needed NSAIDs regularly for pain relief. The AOFAS score improved significantly $(P=0.012)$ from 32.1 prefusion to 71.5 points at 1 year as did the Mazur score, which rose by 31.2 to 72.5 points at 1 year $(P=0.012)$. The SF-36 score improved significantly in the domains physical functioning, role limitations due to physical problems, bodily pain, vitality, social functioning and mental health, as did the Physical Component Summary Score. Retrograde intramedullary nailing for tibiocalcaneal arthrodesis can produce a good outcome. However, in the presence of infection, patient selection for intramedullary procedures must be carefully considered on a case-by-case basis.

Keywords Talectomy - Tibiocalcaneal arthrodesis . Retrograde · Intramedullary nail · Ankle arthrodisis . Hindfoot

\section{Introduction}

Loss of the talus is a rare but devastating condition. It may be caused by trauma, avascular necrosis (AVN) and surgical interventions (talectomy for infection, for tumour or failed ankle joint replacement). Subtotal talectomy involves the loss of the talar dome or the talar body; in total talectomy, the entire talus is lost. The remaining hindfoot must be stabilized to prevent major disability [1-3]. A variety of techniques have been described [4]. These range from complex and invasive 
procedures such as the Blair fusion, [5] which requires an intact head and neck of the talus; prosthetic replacement of the talus; [6] use of block autografts [7] or allografts [8] to less invasive techniques. Günal et al. [9] described the creation of a tibiocalcaneal pseudarthrosis, with lateralisation of the medial malleolus, in order to narrow the ankle mortise and stabilize the hindfoot. To date the most widely used techniques involve the use of screws [10], external fixators [10-13] or plates [14]. The advantages of intramedullary devices are that they provide good primary stability and cause limited soft-tissue damage.

We performed a prospective study to assess the 1-year results of tibiocalcaneal arthrodesis using a latest-generation fixed-angle intramedullary nail.

\section{Materials and methods}

In the period 2006-2007, nine tibiocalcaneal arthrodeses were performed at two tertiary-level hospitals. Institutional review board approval and informed consent from each patient had been obtained prior to the study.

There were six men and three women; the mean patient age was 55 years (range: $36-75$ years). Three patients had complete loss of the talar body and five patients subtotal loss where the talar head was preserved. One had lost the entire talus (Fig. 1) (Table 1). Five patients had chronic osteomyelitis. One severely obese (220 kg bodyweight) patient with polyneuropathy (Patient 3; Table 1) had suffered recurrent ankle sprains resulting in AVN of the talus. A neuropathic joint (Charcot) and infection were ruled out previously. Two patients (Patient 2 and Patient 5; Table 1) had AVN of the talus as a result of severe arthrosis with one of these patients (Patient 5; Table 1) sustaining an atraumatic fracture of the talus. The last patient had necrosis of the talus after a fracture (Patient 7; Table 1).

The tibiocalcaneal arthrodesis was performed using a retrograde intramedullary nail $\left(\mathrm{T} 2{ }^{\mathrm{TM}}\right.$ Ankle Arthrodesis Nail AAN; Stryker Trauma GmbH, Schönkirchen/Kiel, Germany). This device features a distal $5^{\circ}$ right or left lateral (valgus) bend to enhance the fit through the calcaneus. The nails come in 10, 11 and $12 \mathrm{~mm}$ diameters and in 150, 200 and $300 \mathrm{~mm}$ lengths. The driving end (distal portion when implanted) diameter is $12 \mathrm{~mm}$ for all nails. The nail design provides for distal fixation with a talar screw and one lateral and one posterior calcaneal screw, to create a fixed-angle construct. The nails may be used with static or dynamic compression, or without compression.

In all cases, a transfibular approach was used. In eight patients the lateral malleolus was removed; in the obese patient (Patient 3; Table 1; Fig. 2), the lateral malleolus was re-attached after resection of the articular surface. The joint surfaces were thoroughly debrided and any non-viable bone completely removed. In patients with infection-related talar loss, much of the talus had been previously removed. In these patients, bacterial cultures that were taken in previous operations were found negative. Where the neck or the head of the talus had been preserved, these structures were apposed to the tibia but not fixed. The cortex of the anterior tibia was not removed. Once contact between viable bone in the tibia

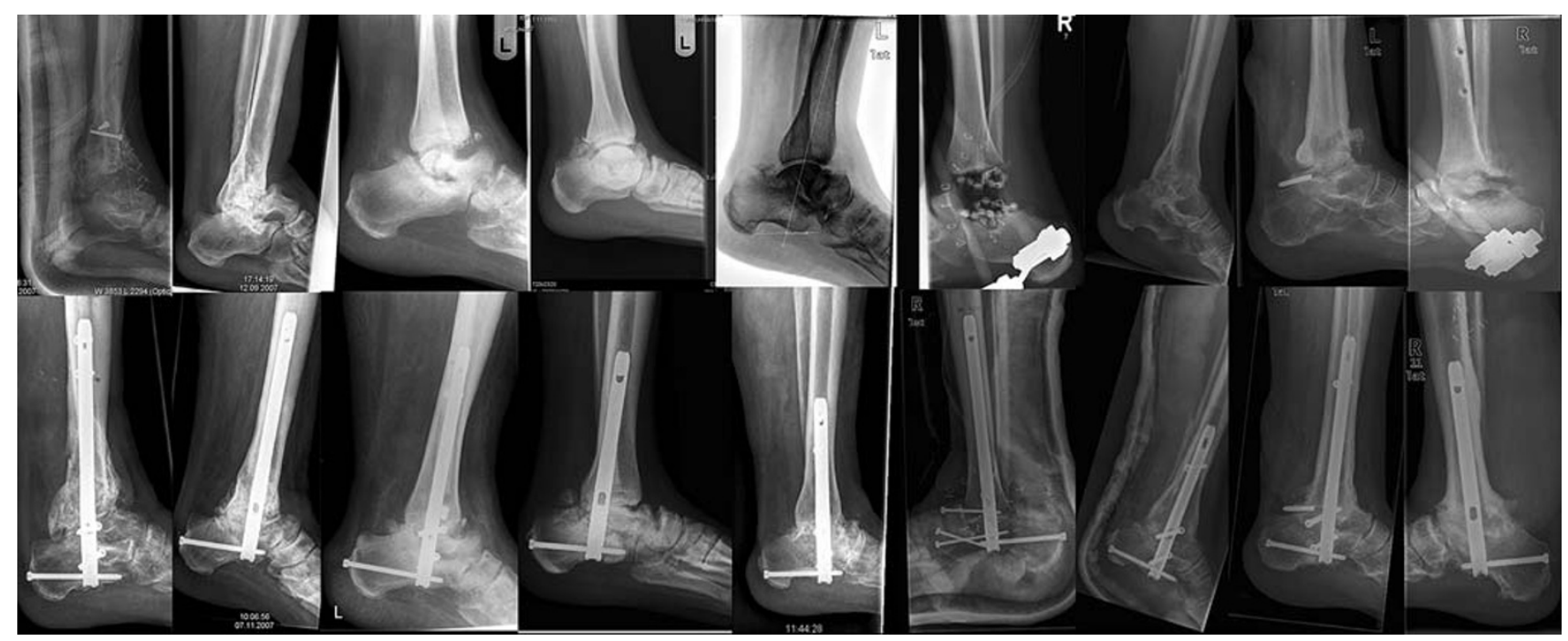

Fig. 1 Preoperative (top row) and postoperative (bottom row) lateral radiographs of the patients (from left to right: Patients 1 through 9). For patient details, see Table 1 


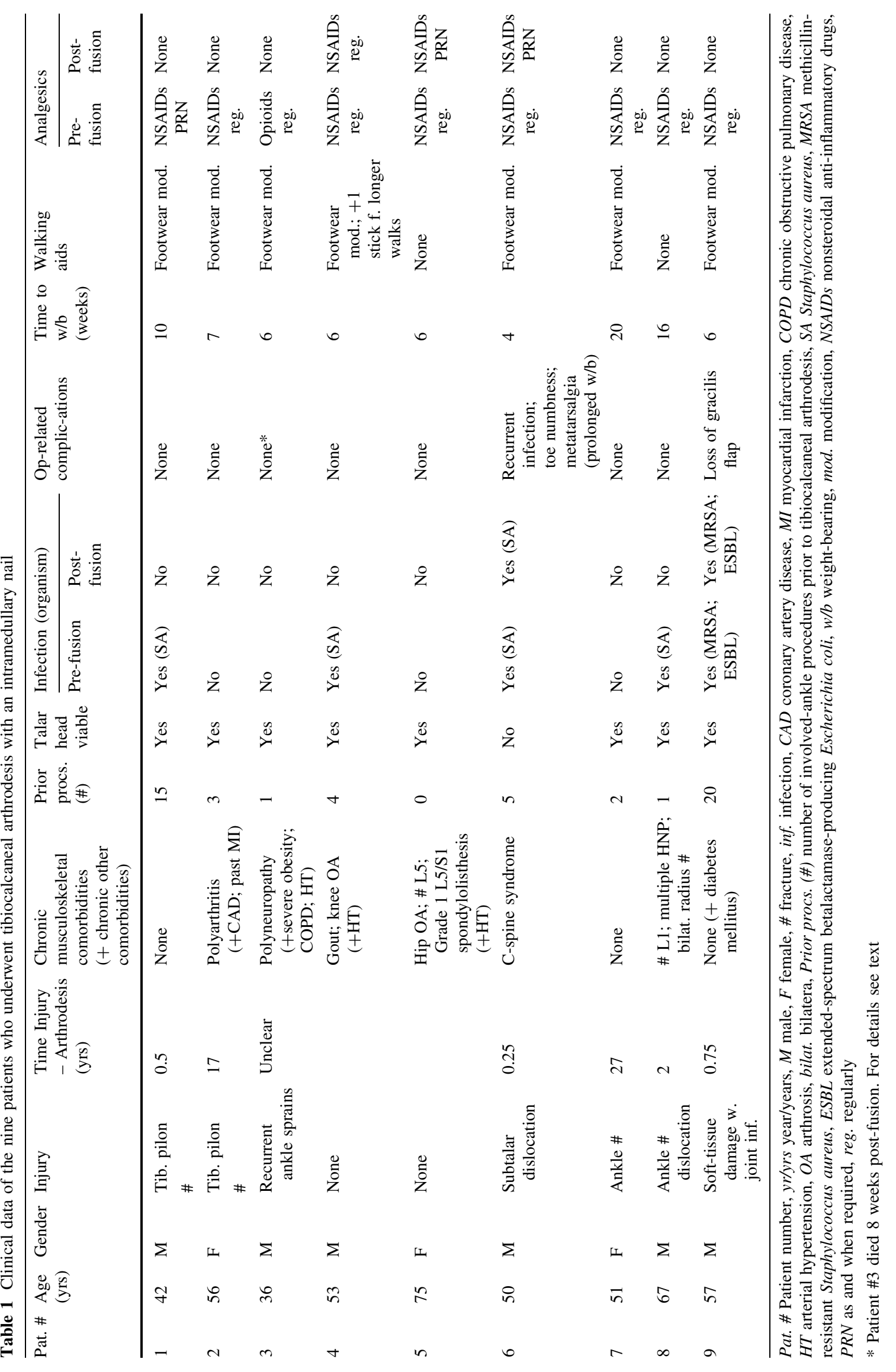



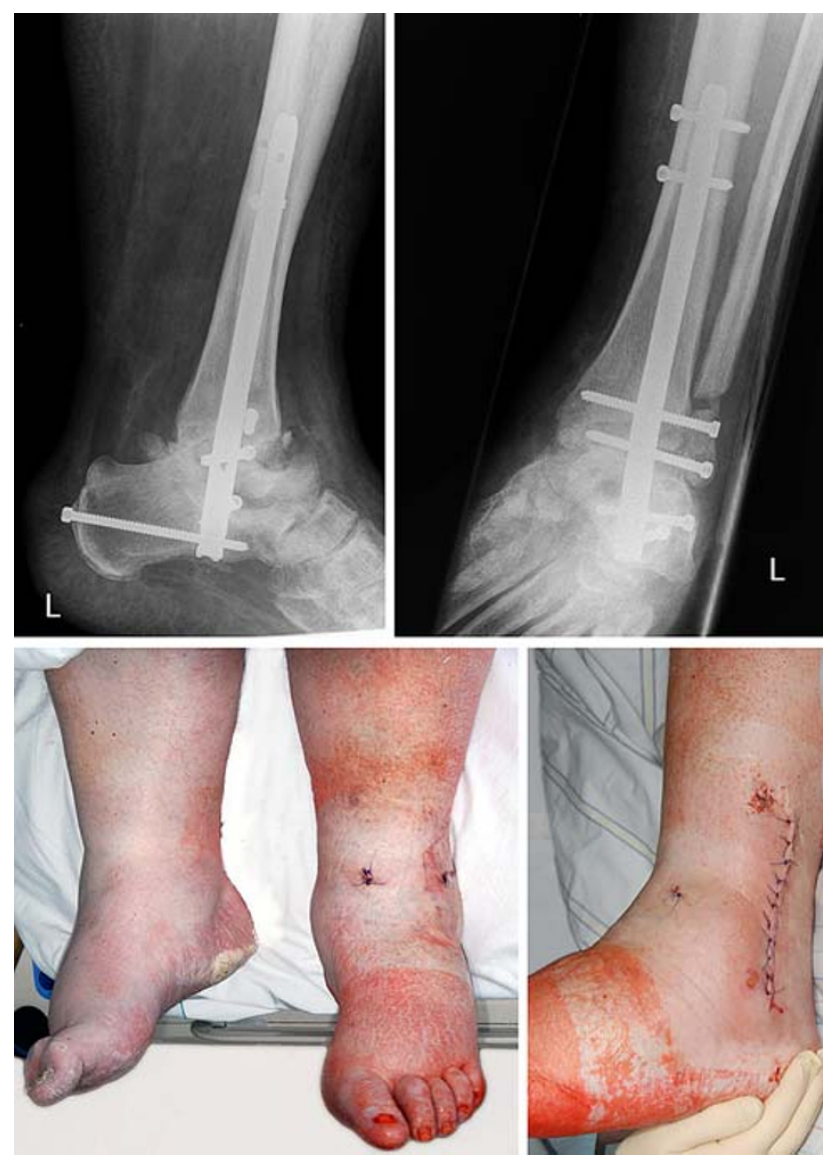

Fig. 2 Six-week radiographs (left) and appearance of the foot immediately post-fusion (right), of Patient 3. For patient details, see Table 1

and the calcaneus had been obtained, intramedullary nail arthrodesis was performed. Since internal compression through the talar locking screw that forms part of the nail system could not be used, patients were managed either with external compression through the calcaneus only, or with impaction of the fusion site using controlled tapping of the intramedullary nail following distal interlocking. In one patient with total loss of the talus, the tibionavicular interval and the calcaneocuboid joints were fused with screws additionally. Finally, a cancellous autograft from the iliac crest or, where possible, from the resected lateral malleolus, was placed across the fusion site. A splint was applied until the soft tissues had healed. After wound healing, five patients were prescribed an FP Walker ${ }^{\mathrm{TM}}$ (Aircast, Vista, CA) while four patients had a walking cast applied. Subsequently, all patients were fitted with orthopaedic footwear incorporating a heel-to-toe rocker sole.

The patients were first assessed during their in-patient stay. On admission, the ASA (American Society of Anesthesiologists) physical status grading system [15] was administered. Chronic musculoskeletal, other comorbidities and previous surgical procedures involving the affected ankle were documented. The following information was recorded during the first in-patient episode and 6 weeks, 6 months, and 1 year after the fusion procedure:

1. Anteroposterior and lateral radiographs;

2. patients' satisfaction with the procedure and the outcome;

3. use of analgesics; and

4. health-related quality of life, for which the SF-36 score [16] was used.

At the same time-points, the AOFAS (American Orthopaedic Foot and Ankle Society) ankle-hindfoot rating scale [17] and the Mazur Ankle Arthrodesis Score [18] were obtained. These scores contain subjective and objective components, with an emphasis on pain. The widely used AOFAS awards a maximum of 100 points if the hindfoot range of motion (ROM) is full. Following arthrodesis, the maximum possible number of points is 86 . The Mazur score was developed for the assessment of ankle arthrodesis. This score awards a maximum of 100 point for a full ROM; patients with a fusion could have a maximum score of 90 points. Osseous fusion was defined to be present when trabeculae crossing the site of the arthrodesis were seen radiographically, resulting in the disappearance of the gap.

Statistical analysis was performed using SPSS for Windows Version 16.0.1 (SPSS Inc., Chicago, IL). Normality in data distribution was tested with the ShapiroWilk test. Non-parametric analysis of data from the patients was carried with the Wilcoxon signed-rank test. Significance was set at $P<0.05$.

\section{Results}

All the patients were available for follow-up at 6 weeks. One subject (the severely obese Patient 3 ) died at 8 weeks postoperatively of a pulmonary embolus. This patient had a pre-surgical history of thrombo-embolic events. After his return home, his anticoagulant therapy was discontinued abruptly at 6 weeks because of a suspected small intracerebral haemorrhage. The remaining eight patients were followed up for 1 year and therefore only their results are subject of this study.

Preoperative physical status, previous surgical procedures and chronic comorbidities

The mean ASA score was 2.2 (range: 2-3). The affected ankle had, on average, undergone six (range 1-20) prior procedures. Comorbidities are listed in Table 1. 
Fig. 3 One-year results (walking downstairs), in a selection of patients (Patients 2, 5, and 6). For patient details, see Table 1
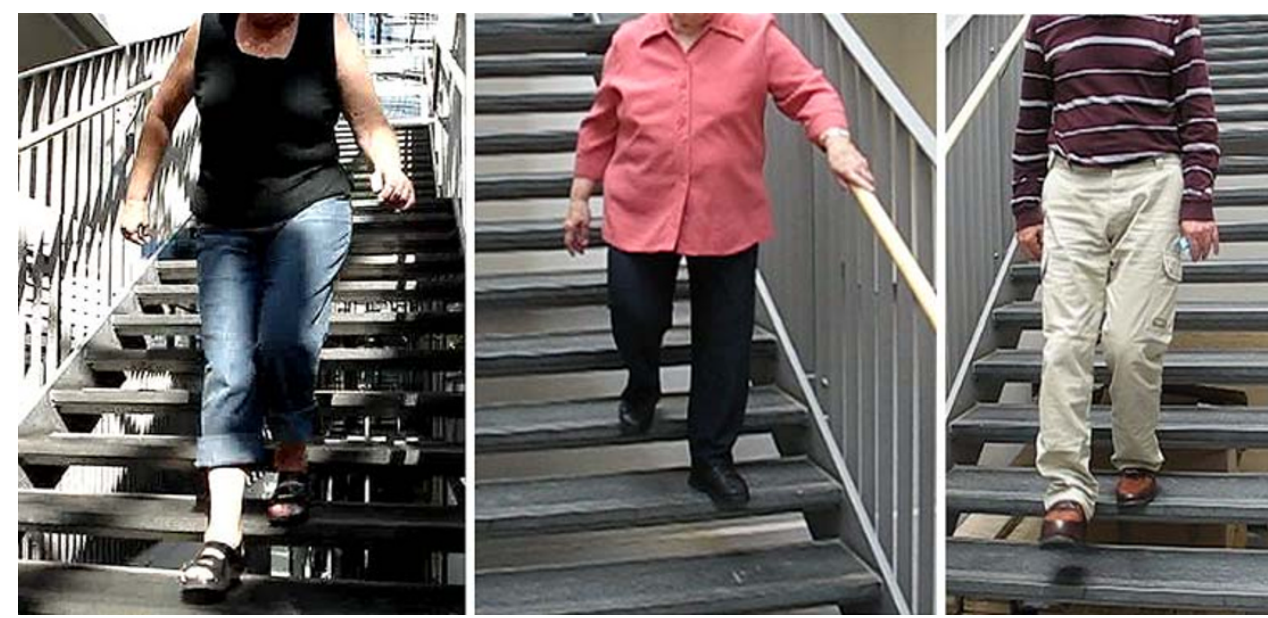

Evidence of bony union and time to weight-bearing

The average time to full weight-bearing was 9 weeks (Fig. 3). Patient 3, who died in Week 8 showed trabeculation across the fusion site at the time of his death, and had resumed full weight-bearing (Fig. 2). Whilst this may suggest union had occurred, the absence of longer term followup had prevented full confirmation. Bony union was assured in all other patients.

\section{Subjective satisfaction}

All the patients were satisfied with the outcome, the postoperative course and the appearance of the operated limb.

\section{AOFAS score}

The mean AOFAS score improved significantly $(P=0.012)$ from 32.1 points pre-fusion to 72.5 points at 1 year post-fusion (Fig. 4).

\section{Mazur Ankle Arthrodesis score}

The mean Mazur score improved significantly $(P=0.012)$ by 32.1 points to 71.5 points at 1 year post-fusion (Fig. 5).

SF-36

The analysis of the SF-36 was based on a German population. At 1 year post-fusion, the mean SF-36 showed significant improvement in the domains physical functioning, role limitations due to physical problems, bodily pain, vitality, social functioning, and mental health, as well as in the Physical Component Summary Score. There were no significant differences in the domains General Health Perception and Role Limitations due to emotional problems, and in the Mental Health Summary Score (Table 2).

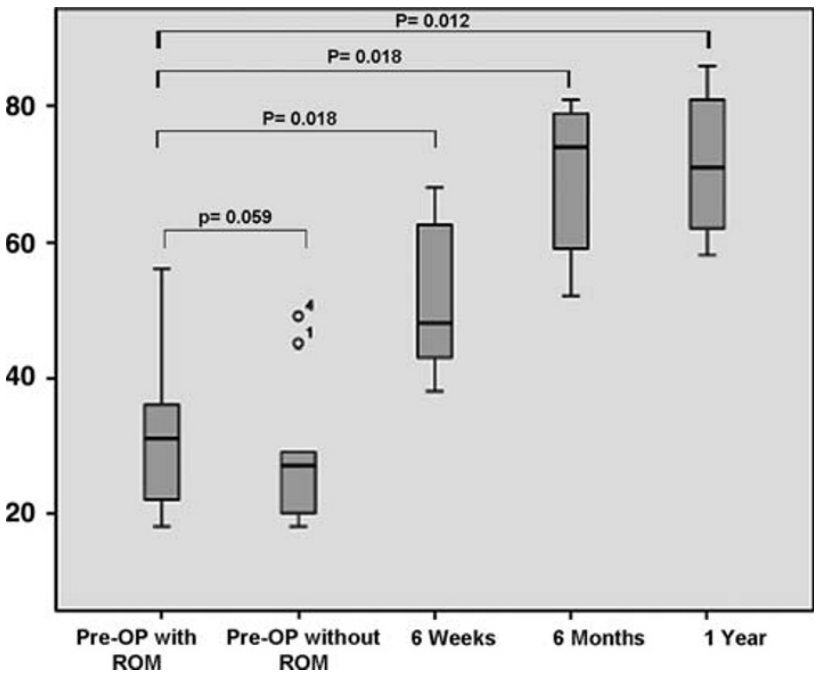

Fig. 4 Pre-fusion versus post-fusion AOFAS ankle-hindfoot scores. Preoperatively, there was no significant difference between the scores with, and those without, range of motion (ROM)

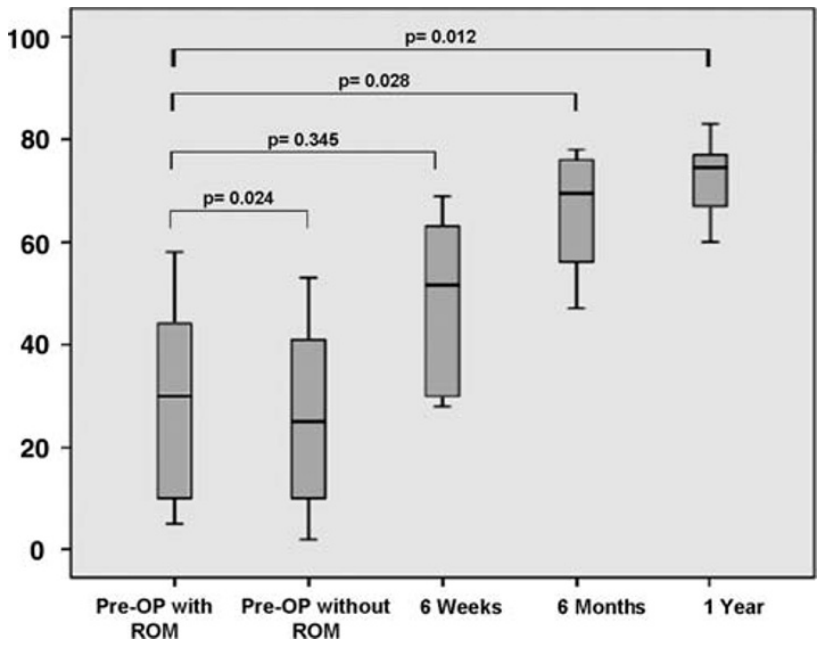

Fig. 5 Pre-fusion versus post-fusion Mazur Ankle Arthrodesis score. There was no significant difference between the preoperative scores with range of motion (ROM) and the scores at 6 weeks 
Table 2 SF-36 domains and component summary scores pre- and 1 year post-fusion

\begin{tabular}{lllr}
\hline Domain/Component score & $\begin{array}{l}\text { Pre-fusion } \\
\text { (mean values and SD) }\end{array}$ & \multicolumn{1}{c}{$\begin{array}{l}\text { At 1 year post-fusion } \\
\text { (mean values and SD) }\end{array}$} \\
\hline Physical functioning & $24.5 \pm 13.2$ & $42.8 \pm 11.2$ & 0.018 \\
Role limitations due to physical problems & $31.7 \pm 11.5$ & $44.2 \pm 8.0$ & 0.028 \\
Bodily pain & $30.6 \pm 14.7$ & $46.5 \pm 11.0$ & 0.018 \\
General health perception & $41.5 \pm 12.5$ & $54.2 \pm 7.7$ & 0.075 \\
Vitality & $38.7 \pm 8.4$ & $49.9 \pm 2.9$ & 0.043 \\
Social functioning & $41.0 \pm 17.4$ & $52.4 \pm 8.4$ & 0.043 \\
Role limitations due to emotional problems & $45.8 \pm 8.8$ & $50.3 \pm 9.0$ & 0.138 \\
Mental health & $43.1 \pm 13.2$ & $52.2 \pm 6.6$ & 0.043 \\
Physical component summary score & $25.6 \pm 17.6$ & $41.8 \pm 9.4$ & 0.043 \\
Mental component summary score & $49.3 \pm 9.6$ & $52.3 \pm 4.5$ & 0.080 \\
\hline
\end{tabular}

\section{Complications}

Complications are listed in Table 1. Patient 3 died from a pulmonary embolism in week 8 after stopping anticoagulants for a suspected intracerebral haemorrhage. One patient (Patient 4) reported heel pain. One patient (Patient 6 ), despite negative intra-operative microbiologic cultures, had a recurrence of infection. This patient had total loss of his talus following repeated surgery and had undergone screw fixation of the tibionavicular and the calcaneocuboid sites additionally, and a peroneus brevis flap. With recurrence of infection, the fixation implants were removed at 1 year post-fusion, and the sinus tract excised and the wound debrided. At that time the fusion site had healed. Five episodes of debridement were needed to obtain a clean wound and the defect was covered with a split-skin graft. There was no evidence of recurrence of infection subsequently but this patient had numbness of the toes and recurrent metatarsalgia on prolonged weight-bearing (Fig. 6). Patient 9 had a softtissue defect that was treated with a gracilis flap at the same time as the index procedure. This flap was lost and the defect was managed with a free latissimus dorsi flap subsequently.
Fig. 6 Appearance of the foot of Patient 6 one year post-fusion
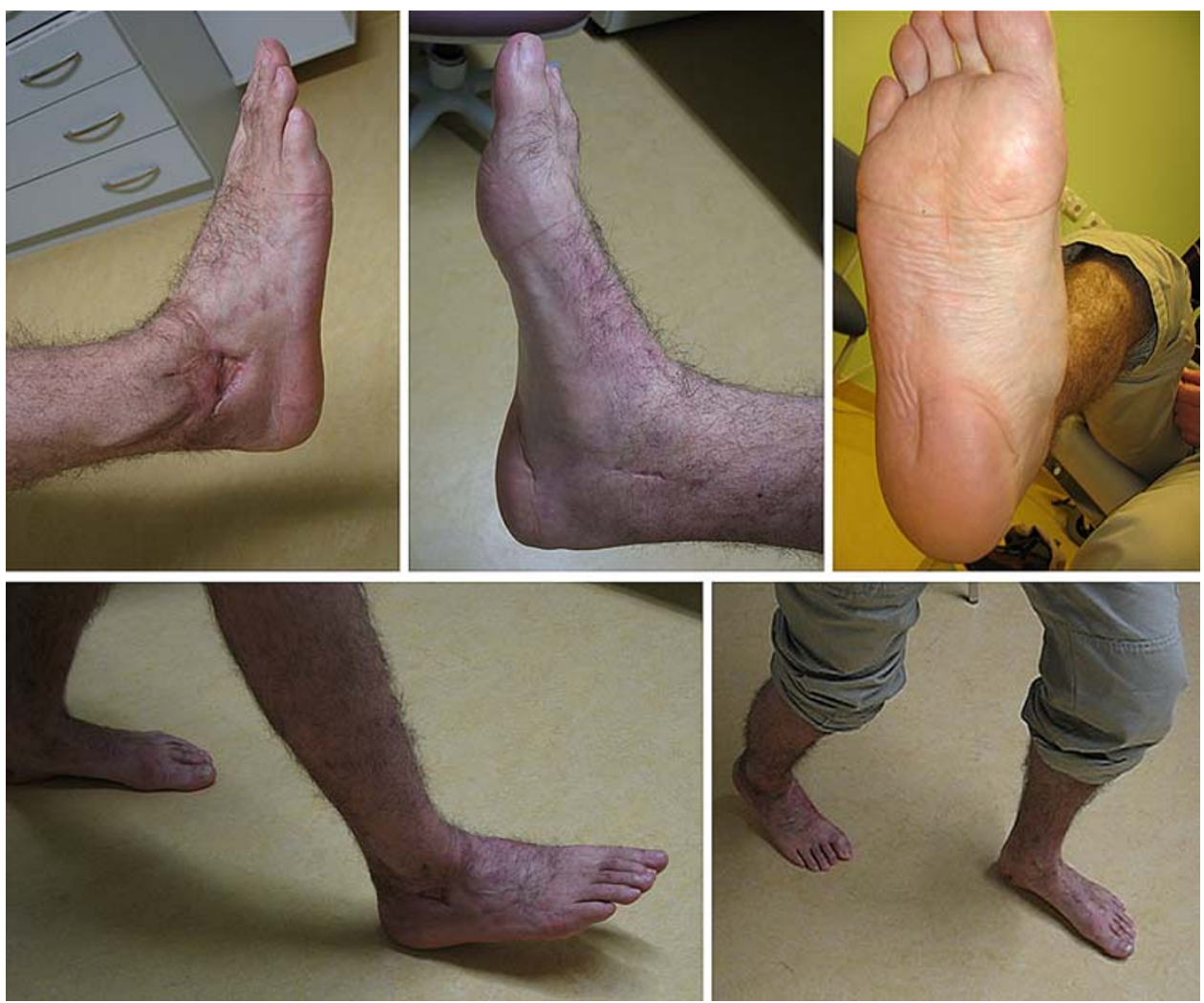
Need for walking aids and analgesics

At one-year, two patients no longer required made-tomeasure orthopaedic footwear. Only one patient (Patient 4; Table 1) required special footwear and a stick when walking longer distances; this patient also had gout and arthrosis of the knee. Patient 4 was the only one requiring regular use of non-steroidal anti-inflammatory drugs (NSAIDs) (Fig. 4). Seven patients had required analgesics on a regular basis before surgery; one of the seven (Patient 4; Table 1) had been on opioids. After surgery, only two patients (Patients 1 and 9; Table 1) required NSAIDs periodically.

\section{Discussion}

In 2000, Myerson et al. [14] reported the results of 30 tibiocalcaneal fusions using a blade plate and screws. There were two nonunions and one plate breakage. There were also two cases of stress fractures of the tibia at the proximal end of the plate. The authors felt an intramedullary device would not provide sufficiently rigid fixation because the calcaneal fragment was relatively small and would allow insertion of only one interlocking screw.

Since then, the design of intramedullary devices has improved. The nail used in this study consistently allows the insertion of two interlocking screws into the calcaneus. Our results suggest that this device provides sufficient stability for tibiocalcaneal arthrodesis.

In 1972, Reckling [3] described a tibiocalcaneal fusion technique using a Charnley compression device. If the head and neck of the talus were intact, these structures were made to abut the anterior tibia. Of the 16 fusions performed, 15 were successful. We have also adopted a policy of preserving the head of the talus, wherever possible, and apposing the head and neck of the talus to the tibia.

Rochman et al. [13] performed 11 tibiocalcaneal arthrodeses using an Ilizarov external ring fixator. Eight patients underwent concomitant lengthening with the Ilizarov fixator. Nine of the 11 patients had successful fusions. At a mean follow-up of 35 months, the mean AOFAS score was 65 [13] and is comparable with our functional results (71.5 points after 1 year). Cancellous bone grafts were not used at the fusion site. Of the seven patients with infected nonunion prior to fusion, none had a recurrence of deep infection in follow-up. The fixator was used for an average of 7.3 months (5.3 months when no lengthening was performed), followed by a 4-6 week period of partial (50\%) weight-bearing [13].

The duration of fixator use reported by these authors differs markedly from the healing time that can be achieved with an intramedullary device. In this study, the stability provided by the construct allowed the patients to resume full weight-bearing after 9 weeks on average. All patients in this study progressed to solid fusion. We think these results show the main advantages of an intramedullary device and cancellous bone grafts compared to an external device.

External fixation is also fraught with the problem of pin track infections. This complication was encountered in all patients by Rochman et al. [13] and by Dennison et al. [11]. However, Rochman et al. [13], Kolker et al. [12] and Liener et al. [10] who treated patients with bone loss and deep talar infection using an external fixator did not observe any recurrence of deep infection whereas there was one relapse in this study. This may suggest that a preferential role for external fixation to intramedullary nails in cases of talar infection.

Some authors $[10,11,13]$ have combined tibiocalcaneal fusion using an Ilizarov frame with leg lengthening to prevent limb length discrepancy. However, Dennison et al. [11] reported a number of complications which prompted them to advise against lengthening in patients over the age of 60 years. Limb shortening by $3-5 \mathrm{~cm}$ has been reported $[3,10,11,13]$. We feel this discrepancy can be readily compensated by a rocker sole, and have routinely prescribed such a modification as part of our post-fusion regimen in order to promote more natural ambulation.

The technique for the creation of a tibiocalcaneal pseudarthrosis as described by Günal et al. [9] may constitute a minimally invasive alternative to fusion and might allow a wider range of hindfoot movement. However, Papaioannou et al. [18] reported that it took 2 years for a satisfactory result to be obtained. We feel that in our patients, who had several procedures prior to their tibiocalcaneal arthrodesis, this length of time would have been detrimental.

We defined union by identifying trabeculation across the fusion site on X-ray. At the 6 weeks follow-up we saw signs of union in every patient. However, visualising trabeculation across the fusion site on a plain X-ray is not always possible. So having not seen any implant migration or cut-out at the 1 year follow-up is another main criterion to confirm that union was achieved in our study.

In absolute terms, the number of cases in this study is small and the group heterogeneous with no control group. A comparison of our results with those in the literature is difficult; most of the papers by other authors are retrospective studies, in small and heterogeneous groups, and collected over extended periods of time. The results presented in this article should be seen as short term; the longterm results will be the subject of a future paper.

In conclusion, we consider intramedullary nailing for tibiocalcaneal arthrodesis to be a minimally invasive internal-fixation technique that promises a good outcome in 
a comparatively short time. We conclude that where there is loss of the talus without infection, this technique is superior to external fixation and plate techniques. Recently developed antibiotic-coated intramedullary devices may enhance the safety of nail arthrodesis in cases of infection.

\section{References}

1. Coltart WD (1952) Aviator's astragalus. J Bone Joint Surg [Br] 34:545-566

2. Penny JN, Davis LA (1980) Fractures and fracture-dislocations of the neck of the talus. J Trauma 20:1029-1037

3. Reckling FW (1972) Early tibiocalcaneal fusion in the treatment of severe injuries of the talus. J Trauma 12:390-396

4. Ware JE, Sherbourne CD (1992) The MOS 36-item short-form health survey (SF-36). Med Care 30:473-483

5. Blair HC (1943) Comminuted fractures and fracture dislocations of the body of the astragalus. Am J Surg 59:37-43

6. Harnroongroj T, Vanadurongwan V (1997) The talar body prosthesis. J Bone Joint Surg [Am] 79:1313-1322

7. Koller H, Assuncao A, Kolb K, Holz U (2007) Reconstructive surgery for complete talus extrusion using the sandwich block arthrodesis: a report of 2 cases. J Foot Ankle Surg 46:493-498

8. Thomason K, Eyres KS (2008) A technique of fusion for failed total replacement of the ankle: Tibio-allograft-calcaneal fusion with a locked retrograde intramedullary nail. J Bone Joint Surg [Br] 90:885-888
9. Günal I, Atilla S, Araç S, Gürsoy Y, Karagözlu H (1993) A new technique of talectomy for severe fracture-dislocation of the talus. J Bone Joint Surg [Br] 75:69-71

10. Liener UC, Bauer G, Kinzl L, Suger G (1999) Die tibiokalkaneare Arthrodese. Eine Analyse von 21 Fällen. Unfallchirurg 102:848-854

11. Dennison MG, Pool RD, Simonis RB, Singh BS (2001) Tibiocalcaneal fusion for avascular necrosis of the talus. J Bone Joint Surg [Br] 83:199-203

12. Kolker D, Wilson MG (2004) Tibiocalcaneal arthrodesis after total talectomy for treatment of osteomyelits of the talus. Foot Ankle Int 25:861-865

13. Rochman R, Jackson Hutson J, Alade O (2008) Tibiocalcaneal arthrodesis using the Ilizarov technique in the presence of bone loss and infection of the talus. Foot Ankle Int 29:1001-1008

14. Myerson MS, Alvarez RG, Lam PW (2000) Tibiocalcaneal arthrodesis for the management of severe ankle and hindfoot deformities. Foot Ankle Int 21:643-650

15. Saklad M (1941) Grading of patients for surgical procedures. Anesthesiology 2:281-284

16. Kitaoka HB, Alexander IJ, Adelaar RS, Nunley JA, Myerson MS, Sanders M (1994) Clinical rating systems for the ankle-hind foot, midfoot, hallux and lesser toes. Foot Ankle Int 15:349-353

17. Mazur JM, Schwartz E, Simon SR (1979) Ankle arthrodesis. Long-term follow-up with gait analysis. J Bone Joint Surg [Am] 61:964-975

18. Papaioannou NA, Kokoroghiannis CG, Karachalios GG (1998) Traumatic extrusion of the talus (missing talus). Foot Ankle Int 19:590-593 